\title{
NUMERICAL SOLUTIONS TO CERTAIN STOCHASTIC DIFFERENTIAL EQUATIONS BY USING MILSTEIN METHOD
}

\author{
Adel S. Hussain* \\ Dept. of IT, Amedey Institute, Duhok Polytechnic University, Kurdistan Region-Iraq
}

\section{ABSTRACT}

(Received: April 17, 2018; Accepted for Publication: July 9, 2018)

In this paper, we studied the numerical method to solve the stochastic differential equations. The Milstein method was used because of the difficulty of finding analytical solutions for many of Stochastic differential equations. Numerical simulations for different selected examples are implemented. In addition, the strong convergence error, are supported by numerical tests problems.

KEYWORDS: Numerical Solutions, Certain Stochastic Differential Equations, Milstein method, strongly convergence

for solving stochastic differential equations. This method is known as the Milstein method. $[3,6,9,10]$ proved an application of the central difference and predictor methods for finding a solution of differential equations with stochastic inputs. Numerical methods for SDE's constructed by translating a deterministic numerical method (like the Euler method or Runge-Kutta method,[11,12]).Applying it to a stochastic ordinary differential equation. However, merely translating a deterministic numerical method and applying it to an SDE will generally not provide accurate methods $[2,5,8]$.

\section{Definition 1.[6]}

Let $x(t) \in(0 \leq t \leq T)$ be a stochastic process such that for any $0 \leq t_{1}<t_{2} \leq \mathrm{T}$, we have

$x\left(t_{2}\right)-x\left(t_{1}\right)=\int_{t_{1}}^{t_{2}} a(t) d t+\int_{t_{1}}^{t_{2}} b(t) d w_{1}(t)$

where $a \in \mathrm{L}_{\omega}^{1}[0, \mathrm{~T}], \mathrm{b} \in \mathrm{L}_{\omega}^{2}[0, \mathrm{~T}]$. Also we say that $\mathrm{x}(\mathrm{t})$ has stochastic differential $\mathrm{dw}_{1}$, on $[0, \mathrm{~T}]$, given by $d x(t)=a(t) d t+b(t) d w_{1}(t)$.

\section{Theorem .1.[7].}

Let $d \xi(t)=a d t+b d w(t)$, and let $f(x, t)$ be a continuous function in $(x, t) \in \mathrm{R}^{1} \times[0, \infty)$ together with its partial derivatives $f_{x}, f_{x x}, f_{t}$. Then the process $f(\xi(t), t)$ has a stochastic differential given by

$$
\begin{aligned}
& \operatorname{df}(\xi(t), t)=\left[f_{t}(\xi(t), t)+f_{x}(\xi(t), t) a(t)+\frac{1}{2}\right. \\
& \left.f_{x x}(\xi(t), t) b^{2}(t)\right] d t+f_{x}(\xi(t), t) b(t) d w(t) \\
& { }^{*} \text { E-mail: adel-83-1983@yahoo.com }
\end{aligned}
$$

\section{INTRODUCTION}

Qtochastic differential equations (SDEs) have become standard models for financial quantities such as asset prices, interest rates, and their derivatives. Un- like deterministic models such as ordinary differential equations, which have a unique solution for each appropriate initial condition, SDEs have solutions that are continuous-time stochastic processes. Methods for the computational solution of stochastic differential equations are based on similar techniques for ordinary differential equations, but generalized to provide support for stochastic dynamics. In mathematics, the Milstein method is a technique for the approximate numerical solution of a stochastic differential equation. It is named after Grigori N. Milstein who first published the method in 1974.The numerical methods are based on time discrete approximations. Time discrete approximations for both the strong and weak convergence criteria will be presented. Whereas time discrete approximations which satisfy the strong convergence criterion involves the simulation of sample paths at each step of the discretization time, approximations that satisfy the weak convergence criterion involve the approximation of some function of the ltô process such as the first and second moments at a given final time $\mathrm{T}$. Early attempts are made in the area of numerical methods for stochastic differential equations using Euler-Maruyama method. [1,4,3,7] provides an early account for constructing a numerical 1 phethod 
distribution are generated using the Mat lab function rand $\mathrm{n}(1, \mathrm{~N})$ and each of these variables are then multiplied by $\sqrt{\Delta t}$, resulting in the random increments in equation (2). From equation (2),we get

$\Delta \mathrm{w}_{1}=\mathrm{w}_{\mathrm{t} 1}-\mathrm{w}_{\mathrm{t} 0}$ and $\Delta \mathrm{w}_{2}=\mathrm{w}_{\mathrm{t} 2}-\mathrm{w}_{\mathrm{t} 1}$. That is $\Delta \mathrm{w}_{1}$ $+\Delta \mathrm{w}_{2}=\mathrm{w}_{\mathrm{t} 2}-\mathrm{w}_{\mathrm{t} 1}+\mathrm{w}_{\mathrm{t} 1}-\mathrm{w}_{\mathrm{t} 0}=\mathrm{w}_{\mathrm{t} 2}$. Since $\mathrm{t}_{0}=0$ and $\mathrm{w}_{0}=0$. Therefore $\mathrm{w}_{\mathrm{t}_{\mathrm{n}}}=\sum_{\mathrm{j}=1}^{\mathrm{n}} \Delta \mathrm{w}_{\mathrm{j}}$. For more details, see the computational algorithm for generating Brownian motion supported by Mat lab $[4,10,12]$.

Our work, we propose to solve Certain stochastic differential equations, using the Milstein method in2-wiener process. Furthermore we use some examples to show that numerical solutions from different examples are implemented correctly.

\section{MAIN RESULTS}

In this section we can state and prove the Lemma by using theorem 1.[7].

If $d x=a\left(t, x_{t}\right) d t+b\left(t, x_{t}\right) d z_{1}+b\left(t, x_{t}\right) d z_{2}$ ,and let $\mathrm{f}(\mathrm{x}, \mathrm{t})$ is a continuous function in $(\mathrm{x}, \mathrm{t}) \in \mathrm{R}^{1} \times[0, \infty)$ together with its $\mathrm{f}_{\mathrm{x}}, \mathrm{f}_{\mathrm{xx}}, \mathrm{f}_{\mathrm{t}}$. Then the process $f(x(t) d t, t)$ has a stochastic differential given by $d f=f_{t} d t+a f_{x} d t+$ bf $\mathrm{f}_{\mathrm{x}} \mathrm{dz_{1 }}+\mathrm{kf}_{\mathrm{x}} \mathrm{dz_{2 }}+\frac{1}{2} \mathrm{~b}^{2} \mathrm{f}_{\mathrm{xx}} d \mathrm{z}_{1}^{2}+\frac{1}{2} \mathrm{k}^{2} \mathrm{f}_{\mathrm{xx}} d \mathrm{z}_{2}^{2}$ ... (3)

Proof .

let $f(x, t)$ be a twice continuously differential function of $\mathrm{x}$ and $\mathrm{t}$; consider writing the Taylor expansion of
This is equation is called Itô formula. Notice that if $\mathrm{h}(\mathrm{s})$ were continuously differentiable in $\mathrm{s}$, then (by the standard calculus formula for total derivatives) the term $\frac{1}{2} \mathrm{f}_{\mathrm{xx}} \mathrm{b}^{2} \mathrm{dt}$ will not appear.(For the definitions and proof the theorem see $[6,7])$.

\section{GENERATING BROWNIAN MOTION IN MAT LAB.[8]}

The underlying difference between deterministic and probabilistic differential equations is the need to generate the following random increments of the Brownian motion for the SDE:

$\Delta \mathrm{w}_{\mathrm{n}}=\mathrm{w}_{\mathrm{tn}}-\mathrm{w}_{\mathrm{tn}-1}$

For computational purpose, it is necessary to describe the Brownian motion, where $\mathrm{w}_{\mathrm{t}}$ is specified at discrete $\mathrm{t}$ values. Therefore, let $\Delta \mathrm{t}=$ $\mathrm{T} / \mathrm{N}$, for some positive integer $\mathrm{N}$ and for $\mathrm{T}$ on the interval $[0, T]$. From the definition of Brownian motion:

or equivalently:

$$
\Delta \mathrm{w}_{\mathrm{n}}=\mathrm{w}_{\mathrm{tn}}-\mathrm{w}_{\mathrm{tn}-1} \sim \mathrm{N}\left(0, \mathrm{t}_{\mathrm{n}}-\mathrm{t}_{\mathrm{n}-1}\right)
$$

$$
\Delta \mathrm{w}_{\mathrm{n}}=\mathrm{w}_{\mathrm{tn}}-\mathrm{w}_{\mathrm{tn}-1} \sim \sqrt{\mathrm{t}_{\mathrm{n}}-\mathrm{t}_{\mathrm{n}-1}} \mathrm{~N}(0,1)
$$

where $\mathrm{N}(0,1)$ denotes a standard normally distributed random variable with zero mean and variance equal to one. Here $t_{n}-t_{n-1}=\Delta t$ is the variance of the Brownian motion random variable. In Mat lab, the function

rand $\mathrm{n}(1, \mathrm{~N})$ will generate $\mathrm{N}$ random variables from the standard normal distribution. In order to generate a random variable with variance equal to $\Delta \mathrm{t}$, random variables from the standard normal

$$
\begin{aligned}
d f= & f(x(t) d t, t+d t)-f(x(t), t) \\
& =f_{t} d t+f_{x} d x+\frac{1}{2} f_{t t}(d t)^{2}+\frac{1}{2} f_{x x}(d x)^{2}+f_{x t} d x d t
\end{aligned}
$$

Next, we will substitute equation(3) in equation(4), we get

$$
\mathrm{dx}=\mathrm{adt}+\mathrm{bdz}_{1}+\mathrm{kdz}_{2}
$$

$$
\begin{aligned}
= & \mathrm{f}_{\mathrm{t}} \mathrm{dt}+\mathrm{f}_{\mathrm{x}}\left(\mathrm{adt}+\mathrm{bdz}_{1}+\mathrm{kdz}_{2}\right)+\frac{1}{2} \mathrm{f}_{\mathrm{tt}}(\mathrm{dt})^{2}+\frac{1}{2} \mathrm{f}_{\mathrm{xx}}\left(\mathrm{adt}+\mathrm{bdz}_{1}+\mathrm{kdz}_{2}\right)^{2}+\mathrm{f}_{\mathrm{xt}}\left(\mathrm{adt}+\mathrm{bdz}_{1}+\mathrm{kdz}_{2}\right) \mathrm{dt} \\
= & \mathrm{f}_{\mathrm{t}} \mathrm{dt}+\mathrm{a} \mathrm{f}_{\mathrm{x}} \mathrm{dt}+\mathrm{bf}_{\mathrm{x}} \mathrm{dz}_{1}+\mathrm{kf}_{\mathrm{x}} \mathrm{dz} \mathrm{z}_{2}+\frac{1}{2} \mathrm{f}_{\mathrm{tt}}(\mathrm{dt})^{2}+\frac{1}{2} \mathrm{f}_{\mathrm{xx}}\left(\mathrm{a}^{2} \mathrm{dt}^{2}+\mathrm{abdz_{1 }} \mathrm{dt}+\mathrm{a} \mathrm{kdz}_{2} \mathrm{dt}+\mathrm{b}^{2} \mathrm{dz}_{1}{ }^{2}+\right. \\
& \quad \mathrm{abdtdz} 1+\mathrm{bkdz} 1 \mathrm{dz} 2+\mathrm{akdz} 2 \mathrm{dt}+\mathrm{bkdz} 1 \mathrm{dz} 2+\mathrm{k} 2 \mathrm{dz} 22+\mathrm{afxtdt} 2+\mathrm{bfxtdz} 1 \mathrm{dt}+\mathrm{kfxtdz} 2 \mathrm{dt}
\end{aligned}
$$

Now, we take a crucial step, and only keep terms up to order dt using the following Logic. The standard deviation $\mathrm{dz}$ is of order $\sqrt{\mathrm{dt}}$, hence we think terms up to order dt yielding

$$
\mathrm{df}=\mathrm{f}_{\mathrm{t}} \mathrm{dt}+\mathrm{af} \mathrm{f}_{\mathrm{x}} \mathrm{dt}+\mathbf{b f}_{\mathrm{x}} \mathrm{dz_{1 }}+\mathrm{kf}_{\mathrm{x}} \mathrm{dz_{2 }}+\frac{1}{2} \mathrm{~b}^{2} \mathrm{f}_{\mathrm{xx}} \mathrm{dz_{1 }}{ }^{2}+\frac{1}{2} \mathrm{k}^{2} \mathrm{f}_{\mathrm{xx}} \mathrm{dz_{2 }}{ }^{2}
$$


Finally we replace by $\mathrm{dz}_{1}{ }^{2} \& \mathrm{dz}_{2}{ }^{2}$ it's expectation dt which leads to Itô-lemma.

(6)

$d f=\left(f_{t}+a f_{x}+\frac{1}{2} b^{2} f_{x x}+\frac{1}{2} k^{2} f_{x x}\right) d t+b f_{x} d z_{1}+k f_{x} d z_{2}$

which using the operator

$\mathrm{Lf}=\mathrm{af}$

$\mathrm{L}^{0} \mathrm{f}=\mathrm{af}^{\prime}+\frac{1}{2} \mathrm{~b}^{2} \mathrm{f}^{\prime \prime}+\frac{1}{2} \mathrm{k}^{2} \mathrm{f}^{\prime \prime}$

$\mathrm{L}^{2} \mathrm{f}=\mathrm{kf}^{\prime}$

$\mathrm{L}^{1} \mathrm{f}=\mathrm{bf}^{\prime}$

... (7)

We can express (6) as the integral relation:

$$
f\left(x_{t}\right)=f\left(x_{t_{0}}\right)+\int_{t_{0}}^{t} L^{0} f\left(x_{s}\right) d s+\int_{t_{0}}^{t} L^{1} f\left(x_{w 1}\right) d w_{1}+\int_{t_{0}}^{t} L^{2} f\left(x_{w 2}\right) d w_{2}
$$

for all $\mathrm{t} \in\left[\mathrm{t}_{0}, \mathrm{~T}\right]$. When $\mathrm{f}(\mathrm{x})=\mathrm{x}$, we have $\mathrm{L}^{0} \mathrm{f}=\mathrm{a}, \mathrm{L}^{1} \mathrm{f}=\mathrm{b}$ andL $\mathrm{L}^{2} \mathrm{f}=\mathrm{k}$ and (10) reduces to:

$$
x_{t}=x_{t_{0}}+\int_{t_{0}}^{t} a\left(x_{s}\right) d s+\int_{t_{0}}^{t} b\left(x_{s}\right) d w_{1}+\int_{t_{0}}^{t} k\left(x_{s}\right) d w_{2}
$$

In formula (8) is again applied to the function $\mathrm{f}=\mathrm{a}$ and $\mathrm{b}$ and $\mathrm{k}$, In equation (7) the following is obtain

$$
\begin{aligned}
\mathrm{x}_{\mathrm{t}=\mathrm{x}_{\mathrm{t}_{0}}+} & \left.\int_{\mathrm{t}_{0}}^{\mathrm{t}}\left[\mathrm{a}\left(\mathrm{x}_{\mathrm{t}_{0}}\right)+\int_{\mathrm{t}_{0}}^{\mathrm{s}} \mathrm{L}^{0} \mathrm{a}\left(\mathrm{x}_{\mathrm{z}}\right) \mathrm{dz}+\int_{\mathrm{t}_{0}}^{\mathrm{s}} \mathrm{L}^{1} \mathrm{a}\left(\mathrm{x}_{\mathrm{z}}\right) \mathrm{dw}_{1}+\int_{\mathrm{t}_{0}}^{\mathrm{s}} \mathrm{L}^{2} \mathrm{a}\left(\mathrm{x}_{\mathrm{z}}\right) \mathrm{dw}_{2}\right)\right] \mathrm{ds} \\
& +\int_{\mathrm{t}_{0}}^{\mathrm{t}}\left[\int_{\mathrm{t}_{0}}^{\mathrm{t}}\left[\mathrm{b}\left(\mathrm{x}_{\mathrm{t}_{0}}\right)+\int_{\mathrm{t}_{0}}^{\mathrm{s}} \mathrm{L}^{0} \mathrm{~b}\left(\mathrm{x}_{\mathrm{z}}\right) \mathrm{dz}+\int_{\mathrm{t}_{0}}^{\mathrm{s}} \mathrm{L}^{1} \mathrm{~b}\left(\mathrm{x}_{\mathrm{z}}\right) \mathrm{dw}_{1}+\int_{\mathrm{t}_{0}}^{\mathrm{s}} \mathrm{L}^{2} \mathrm{~b}\left(\mathrm{x}_{\mathrm{z}}\right) d w_{2}\right)\right] d \mathrm{k}_{1} \\
& \left.+\int_{\mathrm{t}_{0}}^{\mathrm{t}}\left[\mathrm{k}\left(\mathrm{x}_{\mathrm{t}_{0}}\right)+\int_{\mathrm{t}_{0}}^{\mathrm{s}} \mathrm{L}^{0} \mathrm{k}\left(\mathrm{x}_{\mathrm{z}}\right) \mathrm{dz}+\int_{\mathrm{t}_{0}}^{\mathrm{s}} \mathrm{L}^{1} \mathrm{k}\left(\mathrm{x}_{\mathrm{z}}\right) \mathrm{dw}_{1}+\int_{\mathrm{t}_{0}}^{\mathrm{s}} \mathrm{L}^{2} \mathrm{k}\left(\mathrm{x}_{\mathrm{z}}\right) \mathrm{dw}_{2}\right)\right] \mathrm{dk_{2 }} \\
& =
\end{aligned}
$$

$\mathrm{x}_{\mathrm{t}_{0}}+\mathrm{a}\left(\mathrm{x}_{\mathrm{t}_{0}}\right) \int_{\mathrm{t}_{0}}^{\mathrm{t}} \mathrm{ds}+\mathrm{b}\left(\mathrm{x}_{\mathrm{t}_{0}}\right) \int_{\mathrm{t}_{0}}^{\mathrm{t}} \mathrm{dk}_{1}+\mathrm{k}\left(\mathrm{x}_{\mathrm{t}_{0}}\right) \int_{\mathrm{t}_{0}}^{\mathrm{t}} \mathrm{dk}_{2}+\mathrm{R}$

where

$\mathrm{R}=\int_{\mathrm{t}_{0}}^{\mathrm{t}} \int_{\mathrm{t}_{0}}^{\mathrm{s}} \mathrm{L}^{0} \mathrm{a}\left(\mathrm{x}_{\mathrm{z}}\right) d \mathrm{zds}+\int_{\mathrm{t}_{0}}^{\mathrm{t}} \int_{\mathrm{t}_{0}}^{\mathrm{s}} \mathrm{L}^{1} \mathrm{a}\left(\mathrm{x}_{\mathrm{z}}\right) \mathrm{dw}_{1} \mathrm{ds}+\int_{\mathrm{t}_{0}}^{\mathrm{t}} \int_{\mathrm{t}_{0}}^{\mathrm{s}} \mathrm{L}^{2} \mathrm{a}\left(\mathrm{x}_{\mathrm{z}}\right) \mathrm{dw} \mathrm{w}_{2} \mathrm{ds}+\int_{\mathrm{t}_{0}}^{\mathrm{t}} \int_{\mathrm{t}_{0}}^{\mathrm{s}} \mathrm{L}^{0} \mathrm{~b}\left(\mathrm{x}_{\mathrm{z}}\right) \mathrm{dz} d \mathrm{k}_{1}$

$+\int_{\mathrm{t}_{0}}^{\mathrm{t}} \int_{\mathrm{t}_{0}}^{\mathrm{s}} \mathrm{L}^{1} \mathrm{~b}\left(\mathrm{x}_{\mathrm{z}}\right) \mathrm{dw_{1 }}(\mathrm{z}) d \mathrm{k}_{1}+\int_{\mathrm{t}_{0}}^{\mathrm{t}} \int_{\mathrm{t}_{0}}^{\mathrm{s}} \mathrm{L}^{2} \mathrm{~b}\left(\mathrm{x}_{\mathrm{z}}\right) \mathrm{dw}_{2}(\mathrm{z}) \mathrm{dk}_{1}+\int_{\mathrm{t}_{0}}^{\mathrm{t}} \int_{\mathrm{t}_{0}}^{\mathrm{s}} \mathrm{L}^{0} \mathrm{k}\left(\mathrm{x}_{\mathrm{z}}\right) \mathrm{dz} \mathrm{dk}_{2}$

$+\int_{\mathrm{t}_{0}}^{\mathrm{t}} \int_{\mathrm{t}_{0}}^{\mathrm{s}} \mathrm{L}^{1} \mathrm{k}\left(\mathrm{x}_{\mathrm{z}}\right) \mathrm{dw}_{1}(\mathrm{z}) \mathrm{dk}_{2}+\int_{\mathrm{t}_{0}}^{\mathrm{t}} \int_{\mathrm{t}_{0}}^{\mathrm{s}} \mathrm{L}^{2} \mathrm{k}\left(\mathrm{x}_{\mathrm{z}}\right) \mathrm{dw}_{2}(\mathrm{z}) \mathrm{dk}_{2}$

Repeat this procedure by applying the formula (8) to $\mathrm{f}=\mathrm{L}^{1} \mathrm{~b}$ in equation (10) to obtain

$\mathrm{x}_{\mathrm{t}}=\mathrm{x}_{\mathrm{t}_{0}}+\mathrm{a}\left(\mathrm{x}_{\mathrm{t}_{0}}\right) \int_{\mathrm{t}_{0}}^{\mathrm{t}} \mathrm{ds}+\mathrm{b}\left(\mathrm{x}_{\mathrm{t}_{0}}\right) \int_{\mathrm{t}_{0}}^{\mathrm{t}} \mathrm{dk}_{1}+\mathrm{k}\left(\mathrm{x}_{\mathrm{t}_{0}}\right) \int_{\mathrm{t}_{0}}^{\mathrm{t}} \mathrm{dk}_{2}+\mathrm{L}^{1} \mathrm{~b}\left(\mathrm{x}_{\mathrm{t}_{0}}\right) \int_{\mathrm{t}_{0}}^{\mathrm{t}} \int_{\mathrm{t}_{0}}^{\mathrm{s}} \mathrm{dw}_{1} \mathrm{dk}_{1}+\mathrm{R}$

where

$$
\begin{aligned}
& \mathrm{R}=\int_{\mathrm{t}_{0}}^{\mathrm{t}} \int_{\mathrm{t}_{0}}^{\mathrm{s}} \mathrm{L}^{0} \mathrm{a}\left(\mathrm{X}_{\mathrm{Z}}\right) \mathrm{dzds}+\int_{\mathrm{t}_{0}}^{\mathrm{t}} \int_{\mathrm{t}_{0}}^{\mathrm{s}} \mathrm{L}^{1} \mathrm{a}\left(\mathrm{X}_{\mathrm{Z}}\right) \mathrm{dw}_{1} \mathrm{ds}+\int_{\mathrm{t}_{0}}^{\mathrm{t}} \int_{\mathrm{t}_{0}}^{\mathrm{s}} \mathrm{L}^{2} \mathrm{a}\left(\mathrm{X}_{\mathrm{Z}}\right) \mathrm{dw}_{2} \mathrm{ds}+\int_{\mathrm{t}_{0}}^{\mathrm{t}} \int_{\mathrm{t}_{0}}^{\mathrm{s}} \mathrm{L}^{0} \mathrm{~b}\left(\mathrm{X}_{\mathrm{Z}}\right) \mathrm{dz} \mathrm{dk}_{1} \\
& +\int_{\mathrm{t}_{0}}^{\mathrm{t}} \int_{\mathrm{t}_{0}}^{\mathrm{s}} \int_{\mathrm{t}_{0}}^{\mathrm{z}} \mathrm{L}^{0} \mathrm{~L}^{1} \mathrm{~b}\left(\mathrm{x}_{\mathrm{u}}\right) \mathrm{dudw_{1 }}(\mathrm{z}) \mathrm{dk}_{1}+\int_{\mathrm{t}_{0}}^{\mathrm{t}} \int_{\mathrm{t}_{0}}^{\mathrm{s}} \int_{\mathrm{t}_{0}}^{\mathrm{z}} \mathrm{L}^{1} \mathrm{~L}^{1} \mathrm{~b}\left(\mathrm{x}_{\mathrm{u}}\right) \mathrm{dw}_{\mathrm{u}} \mathrm{dw}_{2} \mathrm{dk}_{\mathrm{s}}+\int_{\mathrm{t}_{0}}^{\mathrm{t}} \int_{\mathrm{t}_{0}}^{\mathrm{s}} \mathrm{L}^{2} \mathrm{~b}\left(\mathrm{x}_{\mathrm{z}}\right) \mathrm{dw}_{2}(\mathrm{z}) \mathrm{dk}_{1} \\
& +\int_{\mathrm{t}_{0}}^{\mathrm{t}} \int_{\mathrm{t}_{0}}^{\mathrm{s}} \mathrm{L}^{0} \mathrm{k}\left(\mathrm{x}_{\mathrm{z}}\right) \mathrm{dzdk_{2 }}+\int_{\mathrm{t}_{0}}^{\mathrm{t}} \int_{\mathrm{t}_{0}}^{\mathrm{s}} \mathrm{L}^{1} \mathrm{k}\left(\mathrm{x}_{\mathrm{z}}\right) \mathrm{dw}_{1}(\mathrm{z}) \mathrm{dk}_{2}+\int_{\mathrm{t}_{0}}^{\mathrm{t}} \int_{\mathrm{t}_{0}}^{\mathrm{s}} \mathrm{L}^{2} \mathrm{k}\left(\mathrm{x}_{\mathrm{z}}\right) \mathrm{dw}_{2}(\mathrm{z}) \mathrm{dk}_{2} \text {. }
\end{aligned}
$$

\section{$\underline{\text { Remrak.1 }}$}

The It $\hat{O}$-Taylor expansion can thus be considered as a generalization of both the Itô formula and the deterministic Taylor formula.

Now we have The Milstein scheme is obtained by considering the first five terms of Taylor expansion of equation (11). It is given by:

$$
x_{t}=x_{t_{0}}+a\left(x_{t_{0}}\right) \int_{t_{0}}^{t} d s+b\left(x_{t_{0}}\right) \int_{t_{0}}^{t} d k_{1}+k\left(x_{t_{0}}\right) \int_{t_{0}}^{t} d k_{2}+L^{1} b\left(x_{t_{0}}\right) \int_{t_{0}}^{t} \int_{t_{0}}^{s} d w_{1} d k_{1}
$$

Use the following formula 


$$
\int_{0}^{\mathrm{t}} \mathrm{w}_{\mathrm{s}}(\omega) \mathrm{dw}_{\mathrm{s}}(\omega)=\frac{1}{2} \mathrm{w}_{\mathrm{t}}^{2}(\omega)-\frac{1}{2} \mathrm{t}
$$

We find that

$$
\int_{\mathrm{t}_{\mathrm{n}}}^{\mathrm{t}_{\mathrm{n}+1}} \int_{\mathrm{t}_{\mathrm{n}}}^{\mathrm{t}} \mathrm{dw}_{1} \mathrm{dk}_{1}=\frac{1}{2}\left\{\left(\Delta \mathrm{W}_{\mathrm{n}}\right)^{2}-\Delta \mathrm{n}\right\}
$$

From equation (7) we get $L^{1} b=b b$. Thus

$$
\mathrm{L}^{1} \mathrm{~b}\left(\mathrm{x}_{\mathrm{t}_{0}}\right) \int_{\mathrm{t}_{0}}^{\mathrm{t}} \int_{\mathrm{t}_{0}}^{\mathrm{s}} \mathrm{dw}_{1} \mathrm{dk}_{1}=\frac{1}{2} \mathrm{bb}^{\prime}\left\{\left(\Delta \mathrm{W}_{\mathrm{n}}\right)^{2}-\Delta \mathrm{n}\right\}
$$

Therefore the Milstein scheme is defined by

$$
\begin{aligned}
Y_{n+1}= & Y\left(\tau_{n}\right)+a\left(\tau_{n}, Y\left(\tau_{n}\right)\right)\left(\tau_{n+1}-\tau_{n}\right)+b\left(\tau_{n}, Y\left(\tau_{n}\right)\right)\left(w_{\tau_{n+1}}-w_{\tau_{n}}\right) \\
& +k\left(\tau_{n}, Y\left(\tau_{n}\right)\right)\left(w_{\tau_{n+1}}-w_{\tau_{n}}\right)+\frac{1}{2} b\left(\tau_{n}, Y\left(\tau_{n}\right)\right) b^{\prime}\left(\tau_{n}, Y\left(\tau_{n}\right)\right)\left\{\left(w_{\tau_{n+1}}-w_{\tau_{n}}\right)^{2}-\left(\tau_{n+1}-\tau_{n}\right)\right\}
\end{aligned}
$$

For brevity, can be written as

$$
\mathrm{Y}_{\mathrm{n}+1}=\mathrm{Y}_{\mathrm{n}}+\mathrm{a} \Delta \mathrm{n}+\mathrm{b} \Delta \mathrm{W}_{\mathrm{n}}+\mathrm{k} \Delta \mathrm{W}_{\mathrm{n}}+\frac{1}{2} \mathrm{bb}^{\prime}\left\{\left(\Delta \mathrm{W}_{\mathrm{n}}\right)^{2}-(\Delta \mathrm{n})\right\}
$$

The term $b^{\prime}$ is the partial derivative of $b$ with respect to $x$.

\subsection{Strong Convergence Criterion:}

1. We saw in the Euler method has strong order of converges $\gamma=1 / 2$ in $E\left|X_{n}-X(T)\right| \leq C \Delta t^{\gamma}$, the method with classical distribution 1. The strong order of Euler method 1 by adding a correction to the random increment that gives Milstein's method. The Itô -Taylor expansion at an appropriate point produces Milstein's method for the stochastic differential equations(13).

$$
\begin{gathered}
X_{j}=X_{j-1}+\Delta t f\left(X_{j-1}\right)+g\left(X_{j-1}\right)\left(W\left(T_{j}\right)-W\left(T_{j-1}\right)\right)+k\left(X_{j-1}\right)\left(w_{1}\left(T_{j}\right)-w_{1}\left(T_{j-1}\right)\right)+ \\
\frac{1}{2} g\left(X_{j-1}\right) g^{\prime}\left(X_{j-1}\right)\left(\left(W\left(T_{j}\right)-W\left(T_{j-1}\right)\right)^{2}-\Delta t, \quad \text { for } j=1,2, \ldots, L\right.
\end{gathered}
$$

\section{Illustration. (With Absolute Error Test and Comparisons):}

The stochastic differential equations Considered by

with initial condition $\mathrm{x}(0)=0$

$$
\left.\begin{array}{l}
\mathrm{dx}=\mathrm{fxdt}+\mathrm{gxdw}_{1}+\mathrm{gxdw}_{2} \\
(0)=0
\end{array}\right\}
$$

The unique solution has the form

$\mathrm{x}(\mathrm{t})=\mathrm{e}^{\int_{0}^{\mathrm{t}} \mathrm{fds}-\frac{1}{2}\left(\mathrm{~g}_{1}{ }^{2}+\mathrm{g}_{2}{ }^{2}\right) \mathrm{ds}+\int_{0}^{\mathrm{t}}\left(\mathrm{g}_{1} \mathrm{dw}_{1}+\mathrm{g}_{2} \mathrm{dw} \mathrm{w}_{2}\right)}$, for $0 \leq \mathrm{t} \leq 1$.

where $f(t)=$ cost; $g_{1}(t)=g_{2}(t)=\operatorname{sint} ; X_{0}=0 ; Y_{0}=1$. The values absolute error at the final time interval $\mathrm{T}=1$; for $\mathrm{R}=1$, can be shown in table (A). As one can see, generating final time error is obtained for sample space $\mathrm{N}=2^{8}$. 
Table (A): Error generated by the explicit Milstein scheme.

\begin{tabular}{llc}
\hline $\mathrm{R}$ & $\mathrm{N}$ & Error at final time \\
\hline 1 & $2^{5}$ & 0.2904 \\
\cline { 2 - 3 } & $2^{6}$ & 1.7081 \\
\cline { 2 - 3 } & $2^{7}$ & 1.4006 \\
\cline { 2 - 3 } & & 0.1732 \\
\hline 2 & $2^{8}$ & 0.8550 \\
\cline { 2 - 3 } & $2^{7}$ & 0.2975 \\
\hline
\end{tabular}

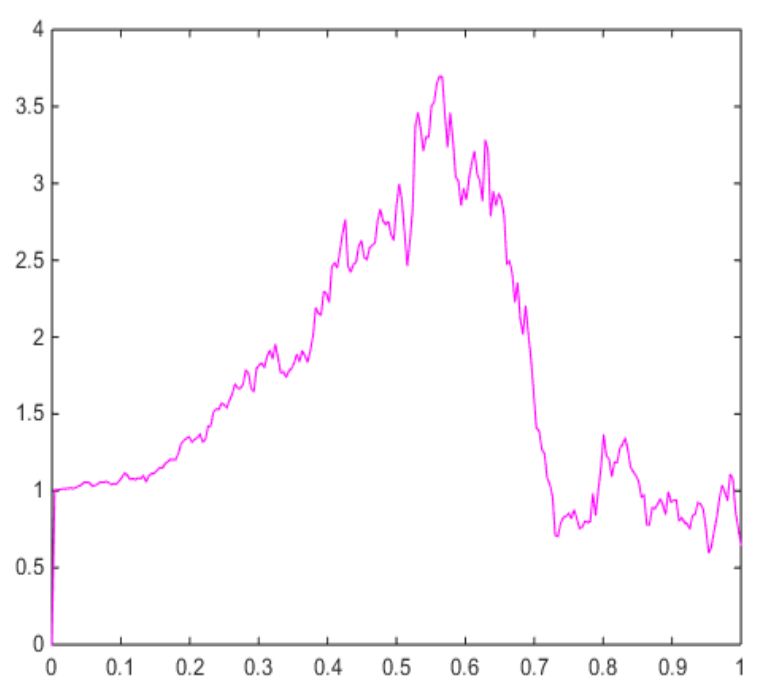

Fig. (1): Exact solution and the numerical solution by Milstein scheme with $N=2^{8} ; \mathrm{R}=1$

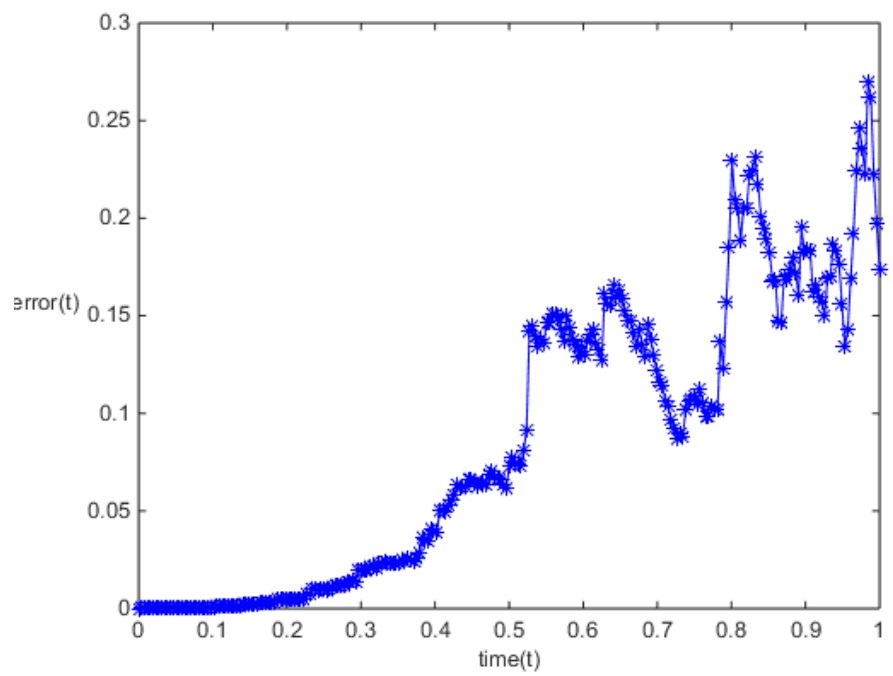

Fig. (1.1): Absolute error between the Euler scheme and exact solution, with $N=2^{8} ; R=1$. 
The absolute error at the final time interval for different sample space numbers, where $\Delta \mathrm{t}=\delta \mathrm{t}$; $\mathrm{R}=1$; the step time for discretization of Brownian motion equals to the step time discretization of explicit Euler scheme are shown in the following (table (B)) and Figure (2.1). As one can see, increasing the sample space generated randomly (N) leads to improving the absolute error at the different time steps, where $\Delta \mathrm{t}=\delta \mathrm{t}$. The Figure (2) show the very good agreement between the exact solution and the corresponding numerical solution
II. Illustration. (With Absolute Error Test and Comparisons):

The stochastic differential equations Considered by

$$
\left.\begin{array}{l}
\mathrm{dx}=\mathrm{g}_{1} \mathrm{xdw}_{1}+\mathrm{g}_{2} \mathrm{xdw}_{2} \\
\mathrm{x}(0)=0
\end{array}\right\}
$$

and the unique solution has the form $x(t)=e^{-\frac{1}{2} \int_{0}^{t}\left(g_{1}{ }^{2}+g_{2}{ }^{2}\right) d s+\int_{0}^{t}\left(g_{1} d w_{1}+g_{2} d w_{2}\right)}$, for $0 \leq \mathrm{t} \leq 1$

where $\mathrm{g}_{1}(\mathrm{t})=\mathrm{g}_{2}(\mathrm{t})=\sin (\mathrm{t}) ; \mathrm{x}_{0}=1 ; \mathrm{y}_{0}=0$.

Table (B): Error generated by the Milstein scheme.

\begin{tabular}{|c|c|c|}
\hline $\mathrm{R}$ & $\mathrm{N}$ & Error at final time \\
\hline \multirow[t]{3}{*}{1} & $2^{5}$ & 0.1360 \\
\hline & $2^{6}$ & 0.7220 \\
\hline & $2^{7}$ & 0.5794 \\
\hline \multirow[t]{3}{*}{2} & $2^{5}$ & 0.8550 \\
\hline & $2^{6}$ & 0.2975 \\
\hline & $2^{7}$ & 0.3392 \\
\hline
\end{tabular}

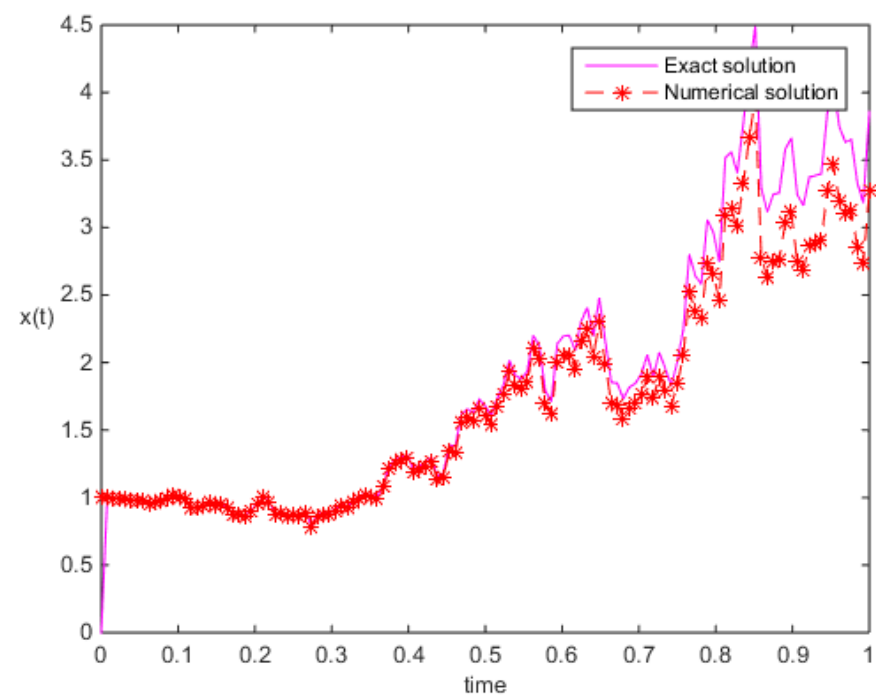

Fig. (2): Exact solution and the numerical solution by Milstein scheme with $\mathrm{N}=2^{5} ; \mathrm{R}=1$. 


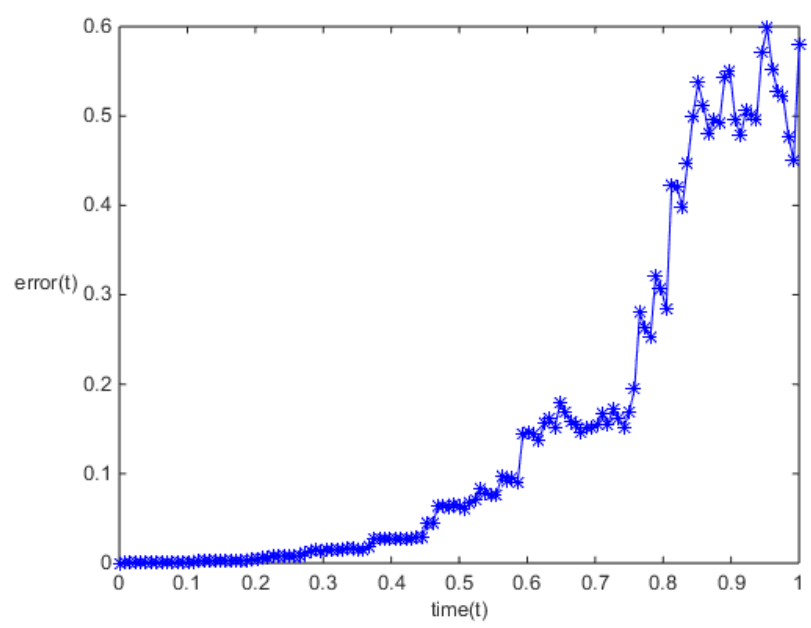

Fig. (2.1) : Absolute error between the Milstein scheme and exact solution with $\mathrm{N}=2^{5} ; \mathrm{R}=1$.

the strong convergence for the numerical solution using Milstein method is obtained. This produces the blue asterisks connected with solid lines in the plot of Figure (3). For reference, a dashed red line of slope one-half is added. The least-squares power law fit gives $q=0.5316$ and reside

\section{Illustration (With Strong Convergence} Test):

The stochastic differential equations Considered by

$\mathrm{dx}=\mathrm{g}_{1} \mathrm{xdw}_{1}+\mathrm{g}_{2} \mathrm{xdw} \mathrm{w}_{2}$

With the condition

$\mathrm{x}(0)=1$

where $g_{1}(t)=g_{2}(t)=\operatorname{sint} ; X_{0}=1 ; N$

$=2^{11} ; \mathrm{T}=1$; sample path $\mu=500$.

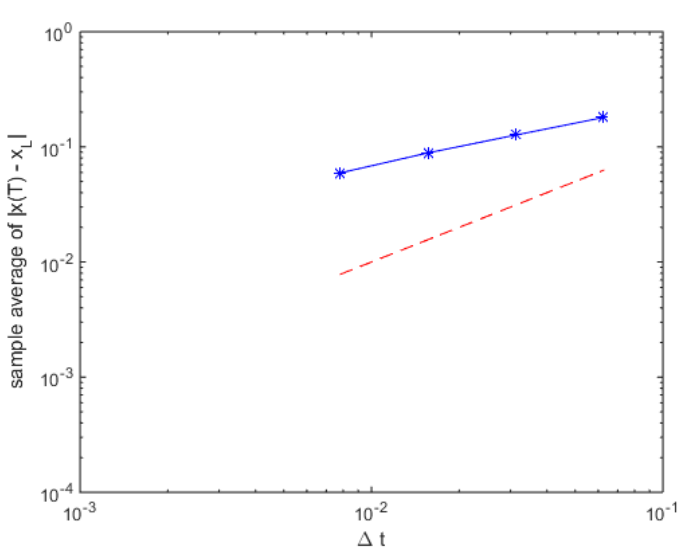

Fig.(3): Milstein strong convergence.

solution based on a method with an analytical approach different from other numerical methods. Finally, this stochastic equation was solved by conventional numerically Milstein method Furthermore we use some examples to show that numerical solutions from different examples are implemented correctly.

\section{REFERENCES}

- Abdu Khaled M., "Mean square stability of secondorder weak numerical methods for stochastic

\section{CONCLUSIONS}

In this paper, we performed a survey on stochastic ordinary differential equations from first-order with time and random coefficients. We indicated a complete analysis for stochastic first order equations in special case of scalar linear order equations. After wards, with making a system of stochastic differential equations from this mentioned equation. it calculated based on the exact solution of this system. We approximated its 
- Fridman, A.F., "Stochastic differential equations and applications",V1, Academic Press, Inc., (1975).

- Gard, T. G. , "Introduction to Stochastic Differential Equations", Marcel Dekker, New York, (1988).

- Kloeden\& Platen, "Numerical Solution of Stochastic Differential Equations",V.23, Applications of Mathematics, New York, Springer-Verlag, Berlin, (1992).

- Romisch W, Winkler R," Step size control for meansquare numerical methods for stochastic differential equations with small noise". SIAM J Sci Comp 28 , (2006).

- Raphael Kruse, Yue Wu, "randomized Milstein method for stochastic differential equations with non-differentiable drift coefficients" ,submitted on 29 Jun 2017 (v1 )31,(2017).

- Tocino A, Ardanuy R ," Runge-Kutta methods for numerical solution of stochastic differential equations".

J Comp Appl Math .,(2004). differential equations", Applied Numerical Mathematics, Vo.48, 127-134,( 2004).

- Arnold J., "Stochastic differential equations; Theory and Applications", John Wiley and Sons, New York, (1974).

- Bernard P. and Fleury G., "Convergence of schemes for stochastic differential equations; Monte Carlo methods", Applied, Vol.7(1), 35-53,( 2001).

- Burrage K, Burrage PM, Tian T., "Numerical methods for strong solutions of stochastic differential equations": an overview. Proc .Roy. Soc .London ,(2004).

- Burrage K. and Burrage P. M., "High strong order explicit Runge-Kutta methods for stochastic ordinary differential equations", Applied Numerical Mathematics, Vol.22, 81-101, (1996).

- Evans, L. C. , "An Introduction to stochastic differential equations", Version 1.2, Lecture Notes, Short Course at SIAM Meeting, July, (2005).

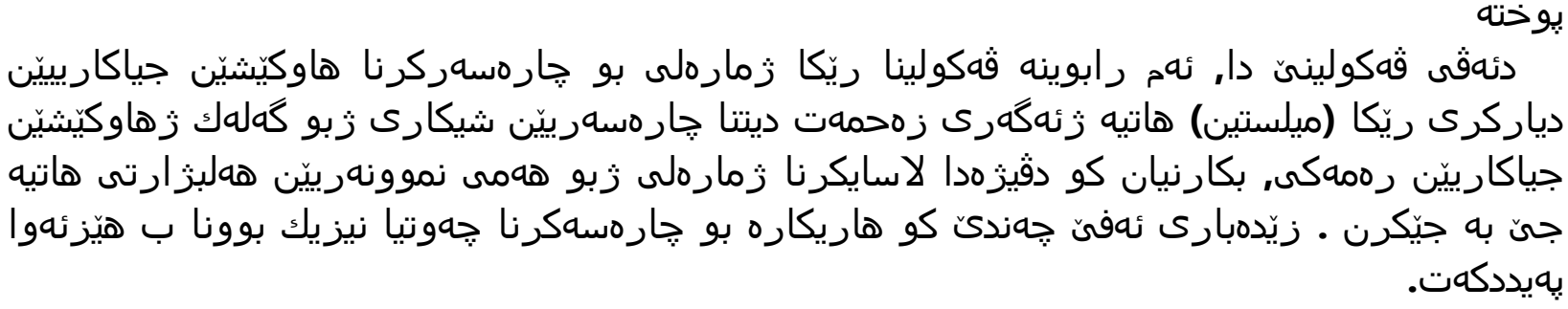

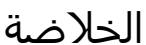

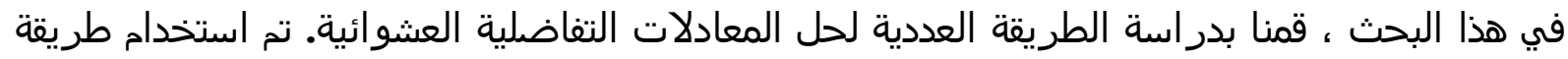

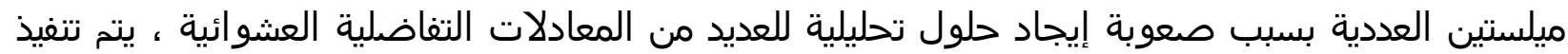

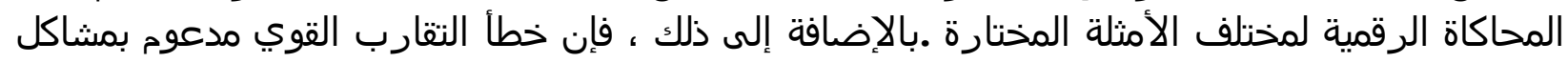
الاختبارات العددية. 